\title{
Tratamiento quirúrgico de fracturas de cóndilo mandibular: Reducción asistida por endoscopio como complemento al tratamiento convencional
}

\author{
Marcelo Mardones M. ${ }^{1,2}$, Coral Torres M. ${ }^{1}$, Renato Gunckel M. ${ }^{1,2}$ y Rodrigo Bravo A. ${ }^{1,2}$
}

\section{Surgical treatment of mandibular condyle fractures: Endoscope-assisted reduction as a complement to conventional treatment}

Introduction: Condyle fractures have a high incidence within jaw fractures. They are mainly treated by open reduction and internal fixation with osteosynthesis elements (ORIF), or by closed reduction (CR) with maxillomandibular fixation (MMF). Endoscopic-assisted treatment of these fractures offers an alternative and surgical complement to the limitations that can occur in classic ORIF. Aim: To describe the technique of endoscope-assisted surgical reduction using transoral access and retromandibular access, as a complement to the conventional surgical technique for the treatment of mandibular condyle fractures, evaluating clinical criteria in a series of cases operated by this technique. Materials and Method: Descriptive study of case series presentation of patients with condyle fracture, treated with endoscopic assisted surgical reduction by transoral and retromandibular access, between the years 2017 and 2018. Results: Of the seven patients operated on, $85.7 \%$ presented normal jaw function, $100 \%$ presented normal facial neurological motor function, and $71.4 \%$ presented no postoperative pain at the 6-month control. All patients recovered the occlusion they had prior to the mandibular fracture. There were no reported cases in need of surgical reintervention. Conclusions: Endoscope-assisted surgical reduction for mandibular condyle fractures is a technique that offers a complement to the conventional surgical technique, allowing greater visibility of the structures, less surgical morbidity and minimal complications in relation to the conventional techniques described.

Key words: mandibular condylar fracture; endoscopic assisted; reduction and internal osteosynthesis.

\section{Resumen}

Introducción: Las fracturas de cóndilo presentan una alta incidencia dentro de las fracturas de mandíbula. Son principalmente tratadas por reducción abierta y fijación interna mediante elementos de osteosíntesis (ORIF) o por reducción cerrada (CR) con fijación máxilo-mandibular (MMF). El tratamiento asistido por endoscopio de estas fracturas, ofrece una alternativa y complemento quirúrgico a las limitaciones que se pueden presentar en la ORIF clásica. Objetivo: Describir la técnica de reducción quirúrgica asistida por endoscopio mediante acceso transoral y acceso retromandibular, como complemento a la técnica quirúrgica convencional para el tratamiento de fracturas de cóndilo mandibular, evaluando criterios clínicos en una serie de casos operados por esta técnica. Materiales y Método: Estudio descriptivo de presentación de serie de casos de pacientes con fractura de cóndilo, tratados con reducción quirúrgica asistida por endoscopio mediante accesos transoral y retromandibular, entre los años 2017 y 2018 . Resultados: De los siete pacientes operados, un $85,7 \%$ presentó una función mandibular normal, un $100 \%$ presentó una función motora neurológica facial normal y un 71,4\% no presentó dolor posoperatorio en el control de los 6 meses. Todos los pacientes recuperaron la oclusión que tenían de forma previa a la fractura mandibular. No se reportaron casos que tuvieran la necesidad de reintervención quirúrgica. Conclusiones: La reducción quirúrgica asistida por endoscopio para las fracturas de cóndilo mandibular es una técnica que ofrece un complemento a la técnica quirúrgica convencional, permitiendo mayor visibilidad de las estructuras, menor morbilidad quirúrgica y complicaciones mínimas en relación a las técnicas convencionales descritas.

Palabras clave: fractura cóndilo mandibular; asistencia endoscópica; reducción y osteosíntesis interna.
'Equipo de Cirugía y Traumatología Maxilofacial, Hospital San José. Santiago, Chile.

2Departamento de Cirugía Maxilofacial Facultad de Odontología Universidad de Chile. Santiago, Chile.

Recibido 2020-01-28 y aceptado 2020-06-29

Correspondencia a: Dr. Marcelo Mardones M. drmardones@gmail.com 


\section{Introducción}

Las fracturas mandibulares representan un $45 \%$ de todas las fracturas del macizo facial. Dentro de estas, las fracturas de cóndilo tienen una alta incidencia y representan el 25\%-35\% de las fracturas mandibulares ${ }^{1}$.

Los principales métodos de tratamiento para las fracturas condilares son la reducción abierta y fijación interna mediante elementos de osteosíntesis (ORIF) y la reducción cerrada (CR) con fijación máxilo-mandibular (MMF)2.

La reducción abierta con fijación interna (ORIF) es necesaria para la alineación apropiada de los segmentos condilares anatómicos. Clásicamente, la ORIF se logra a través de accesos quirúrgicos externos, como el abordaje retromandibular o preauricular y submandibular. Sin embargo, algunas complicaciones de la técnica quirúrgica convencional como fístulas salivales, paresia parcial o completa del nervio facial, cicatriz facial no estética y sangrado de la arteria maxilar y la vena retromandibular presentan una tasa de incidencia del $4 \%$, lo que si bien es bajo, cuando se presenta, genera grandes dificultades clínicas en los pacientes, lo que limita en cierto modo su amplia aceptación y determina la búsqueda de mejoras en la técnica del procedimiento quirúrgico ${ }^{2-5}$.

Los abordajes intraorales al cóndilo mandibular pueden reducir el riesgo de lesión del nervio facial y eliminar las cicatrices faciales, pero carecen de visibilidad adecuada para una correcta reducción y fijación de las estructuras, además de aumentar el tiempo operatorio.

El tratamiento asistido por endoscopio de las fracturas de cóndilo ofrece una alternativa y complemento quirúrgico a las limitaciones que se pueden presentar en la ORIF clásica ${ }^{3}$. La reducción quirúrgica asistida por endoscopio es una técnica mínimamente invasiva que proporciona una excelente visibilidad de las estructuras comprometidas, facilitando la correcta reducción y alineación de los segmentos, reduciendo la morbilidad del procedimiento quirúrgico ${ }^{4}$.

La técnica de reducción quirúrgica asistida por endoscopio se compone de un abordaje transoral a la rama mandibular por donde se trabaja con el endoscopio, el cual proporciona un adecuado campo visual de las estructuras óseas fracturadas. El procedimiento de exposición de la fractura y reducción requiere de instrumentos especializados y una curva de aprendizaje adecuada del equipo tratante. La etapa de fijación mediante osteosíntesis de la fractura se realiza de forma convencional mediante acceso quirúrgico retromandibular ${ }^{3,6}$.
Es así que este procedimiento de asistencia endoscópica complementa a la ORIF, otorgando ventajas como mejor visualización y exposición de las estructuras fracturadas, ventajas que inciden en una reducción del tiempo operatorio y de la morbilidad quirúrgica ${ }^{6}$.

Aquí se describe la técnica de reducción quirúrgica de fracturas condilares asistida por endoscopio mediante acceso transoral y acceso retromandibular extraoral como complemento a la técnica quirúrgica convencional, en una serie de casos de pacientes del Hospital San José, Santiago de Chile. Los objetivos secundarios corresponden a la evaluación de la dinámica mandibular, condición neurológica motora y dolor posoperatorio a los 12 días, 3 meses y 6 meses, así como registrar otros elementos.

\section{Materiales y Método}

Para la recolección de los datos se accedió a la ficha y protocolo operatorio a través de la plataforma virtual del hospital y el Sistema de Información de la Red Asistencial (SIDRA). Para el análisis de los datos recolectados, se utilizó el programa Microsoft Excel.

Los pacientes fueron informados mediante un documento del uso de sus datos clínicos como imagenológicos, lo cual debían autorizar mediante su firma.

Este estudio corresponde a una presentación de serie de casos clínicos tipo descriptivo. La población de estudio correspondió a siete pacientes con diagnóstico de fractura de cóndilo mandibular con desplazamiento a medial del segmento fracturado, tratados con la técnica de reducción asistida por endoscopio mediante acceso transoral y retromandibular como complemento a la técnica convencional, desde diciembre de 2017 a diciembre de 2018.

\section{Criterios de inclusión}

Pacientes con fractura mandibular a nivel de cuello y subcondilares, según la clasificación de Ellis ${ }^{7}$. Con desplazamiento a medial del segmento fracturado, con una angulación $>30^{\circ}$ o con pérdida de la dimensión vertical mandibular $>3 \mathrm{~mm}$, tipo III de acuerdo a la clasificación de Bhagol según grado de desplazamiento ${ }^{8}$. Estas fracturas presentaban un tiempo menor o igual a 2 semanas de evolución. Criterios de exclusión: fracturas de cóndilo sin desplazamiento o $<$ a $30^{\circ}$ y fracturas de cóndilo asociadas a otras fracturas faciales que alteraran la oclusión.

Los pacientes fueron sometidos a exámenes 
clínicos e imagenológicos por medio de tomografía computarizada (TC) (Figuras 1 y 2).

Mediante la técnica que se describe fueron operados siete pacientes con fracturas de cóndilo mandibular con desplazamiento a medial del segmento fracturado, todos fueron tratados mediante reducción quirúrgica asistida por endoscopio y fijación con elementos de osteosíntesis a placas y tornillos.

Todas las cirugías fueron realizadas por el mismo equipo quirúrgico. La técnica empleada se realiza bajo anestesia general, intubación nasotraqueal. Se realiza un abordaje transoral mandibular para lograr un acceso intraoral a la rama mandibular (Figuras 3 A, 4 A y 4 B). Posteriormente, con la ayuda de un endoscopio de óptica $2,1 \mathrm{~mm}$ se identifican la rama y el segmento condilar fracturado.

Luego se procede a reducir o desplazar el segmento fracturado de medial a lateral con el fin de favorecer la fijación mediante osteosíntesis (Figuras 3 B y 3 C). Para realizar esta maniobra, es necesario distraer la rama mandibular hacia caudal, con el fin de generar el espacio suficiente para que el segmento fracturado sea desplazado desde medial hacia lateral. Esta maniobra de tracción mandibular a caudal se consigue con manipulación manual de la mandíbula o con la tracción a través de un alambre de acero quirúrgico lazado a un tornillo de osteosíntesis instalado en el ángulo mandibular (Figura 3 D).

Una vez lograda la reducción del segmento fracturado o, en su defecto, posicionado en la cara lateral de la rama mandibular, se procede a realizar un acceso extraoral retromandibular convencional, para acceder a la rama y segmento condilar fracturado, estructuras que, por las maniobras previas realizadas, su ubicación y exposición es simple de obtener.
Una vez logrado lo anterior se realiza la fijación mediante placas y tornillos de osteosíntesis. (Figura 4 C).

Se registraron el grado de la recuperación en la dinámica mandibular evaluada por el índice de Helkimo ${ }^{9}$, condición neurológica motora facial medida por la escala de House-Brackmann ${ }^{10}$ para evaluación del nervio facial y dolor posoperatorio evaluado a través de la Escala Visual Análoga (EVA) ${ }^{11}$, a los 12 días, 3 meses y 6 meses posoperatorio. Se registraron los tiempos quirúrgicos y de hospitalización posquirúrgico de cada paciente.

El estado de la oclusión dentaria posoperatoria fue evaluado por examen clínico realizado por examinador único que observó si existía máxima intercuspidación dentaria con los dientes presentes dentro de la arcada y con preguntas dirigidas al paciente de si refiere la misma forma de morder previo a su fractura mandibular.

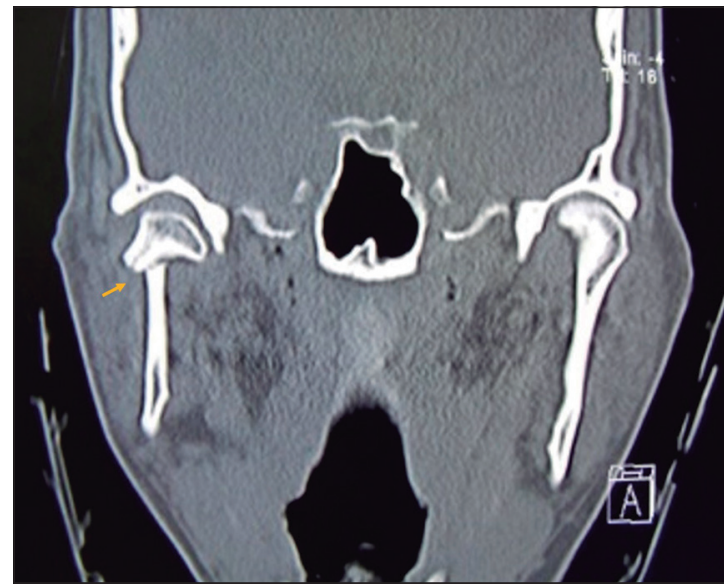

Figura 1. Tomografía computarizada (TC), corte coronal fractura de cóndilo mandibular derecho.

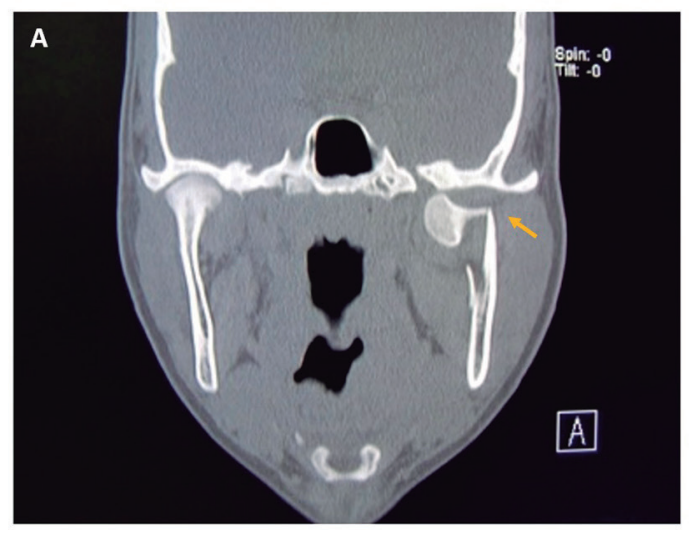

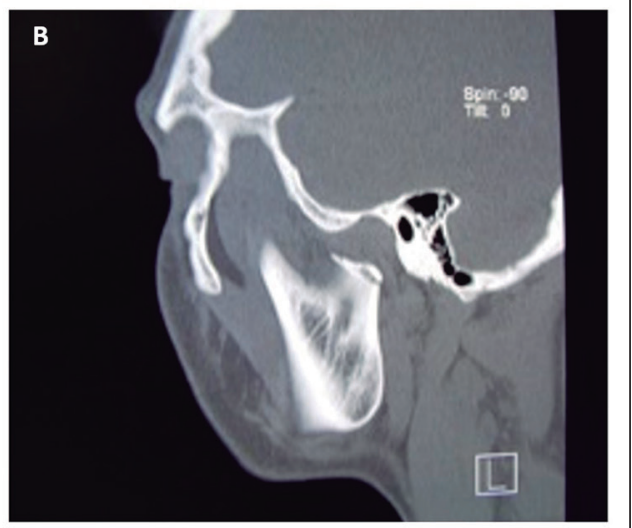

Figura 2. TC fractura de cóndilo mandibular izquierdo. A: Corte coronal, desplazamiento a medial del segmento fracturado. B: Corte sagital, disminución altura vertical de la rama mandibular por desplazamiento a medial del segmento fracturado. 


\section{SERIE DE CASOS}

Figura 3. Vista endoscópica de fractura de cóndilo mandibular derecho. A: Acceso transoral a la rama mandibular derecha. B: Vista medial de la rama mandibular derecha, se observa desplazamiento de segmento condilar fracturado. C: Vista lateral de la rama mandibular, se observa acortamiento de la rama por desplazamiento a la cara medial del segmento fracturado. D: Acceso transparotídeo derecho y amarra alámbrica para tracción mandibular.
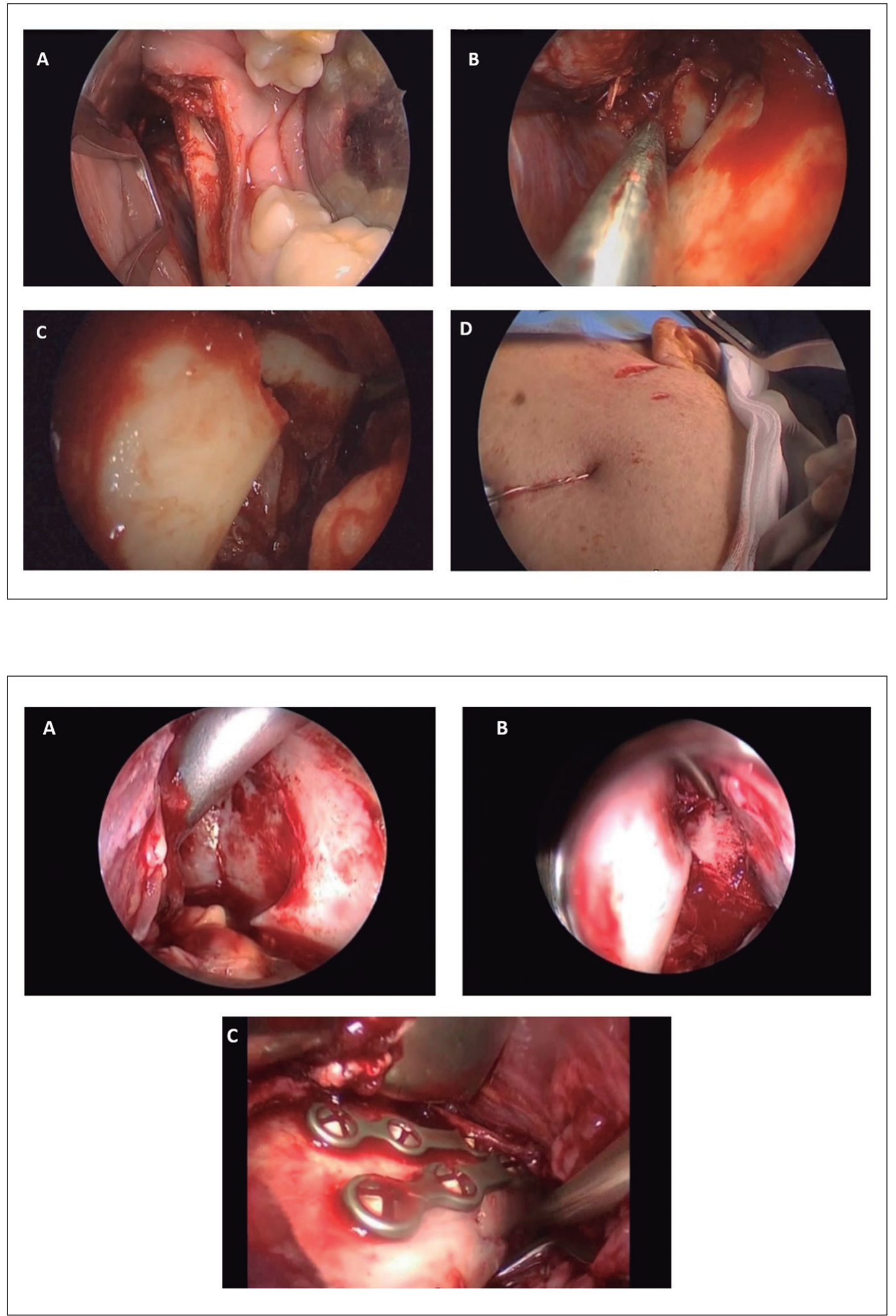

Figura 4. Imagen endoscópica de fractura de cóndilo izquierdo. A: Imagen de la cara medial de la rama mandibular izquierda, se observa língula mandibular y segmento condilar fracturado. B: Vista lateral de la rama mandibular izquierda, se observa segmento condilar fracturado desplazado a la cara medial de la rama. C: Fijación mediante placas y tornillos de osteosíntesis del segmento condilar izquierdo fracturado. 

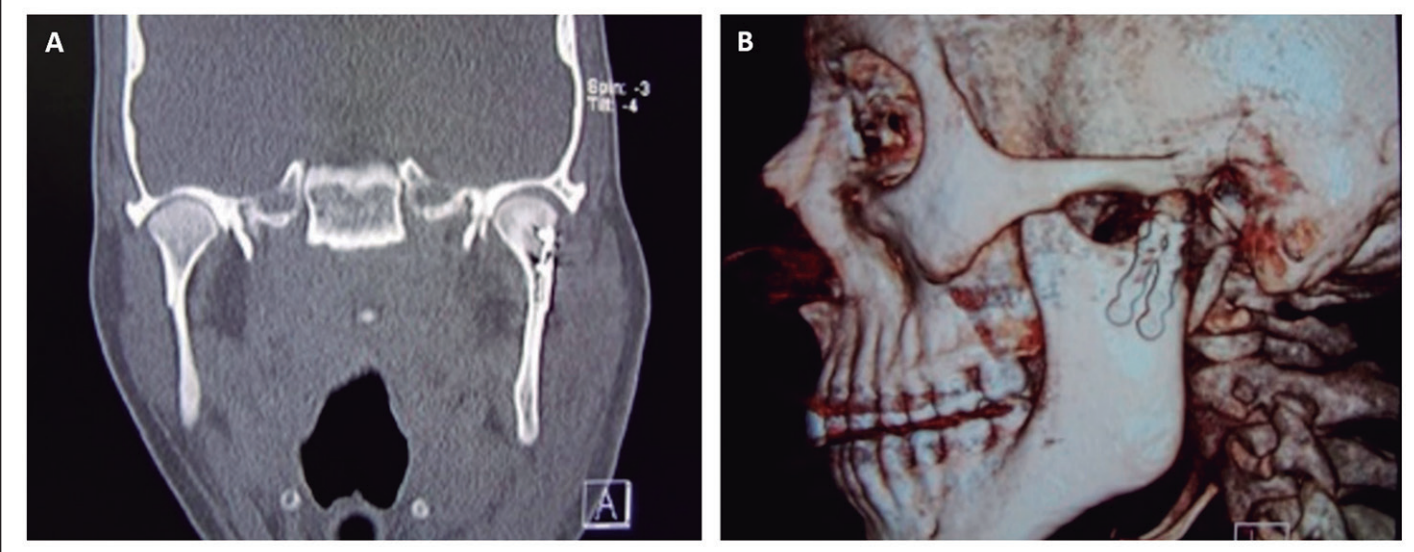

Figura 5. TC de control posquirúrgico. A: Corte coronal, se observa correcta reducción y alineamiento del segmento condilar fracturado del lado izquierdo. B: Reconstrucción 3D, se observan elementos de osteosíntesis para fijación de la fractura del segmento condilar izquierdo.
Además de la evaluación clínica posoperatoria, se indicaron TC posquirúrgicos de control (Figura $5)$.

\section{Resultados}

Fueron operados 7 pacientes, edad media de 35 años (23-58), 4 hombres y 3 mujeres. La etiología del trauma fue agresión por terceros en 3 , caída a nivel en 2 y accidente en bicicleta 2 (Tabla 1).

Los pacientes evolucionaron de forma favorable, con recuperación de la dinámica mandibular, de la oclusión previa al trauma facial y evolución posquirúrgica sin secuelas neurológicas sensitivas ni motoras, tanto en la morbilidad posquirúrgica como en la funcionalidad mandibular.

La funcionalidad mandibular, medida a través del índice de Helkimo ${ }^{9}$, reportó 6/7 con disfunción leve y $1 / 7$ pacientes con disfunción moderada a los 12 días. A los 3 meses $2 / 7$ pacientes presentaron dinámica mandibular normal y $5 / 7$ disfunción leve. Posteriormente, a los 6 meses de operados, 6/7 presentaron dinámica mandibular normal y $1 / 7$ pacientes dinámica mandibular con disfunción leve (Tablas 2, 3 y 8).
La movilidad mandibular (primer criterio del índice de Helkimo) evolucionó de forma favorable, de una apertura bucal prequirúrgica en promedio de $20,6 \mathrm{~mm}$, a un aumento de $32 \mathrm{~mm}, 36,8 \mathrm{~mm}$ y $40,6 \mathrm{~mm}$ a los 12 días, 3 meses y 6 meses posquirúrgico respectivamente (Tabla 4 ).

Con respecto a la evaluación del daño neurológico, medido a través de la escala de HouseBrackmann ${ }^{10}$, 5/7 presentaron una función facial normal (grado I) y $2 / 7$ presentaron una afectación discreta (grado II), principalmente manifestada por la rama bucal del nervio facial durante los primeros 12 días. A los 3 meses, 6/7 presentaron función normal (grado I) y $1 / 7$ presentó una afectación discreta (grado II). Posteriormente, a los 6 meses, todos los pacientes evolucionaron a una función facial normal (grado I) (Tablas 5 y 8).

En referencia al dolor posoperatorio ${ }^{11}$, se reportó $1 / 7$ con dolor leve y $6 / 7$ con dolor moderado a los 12 días, 6/7 con dolor leve y 1/7 con dolor moderado a los 3 meses, y 5/7 de los pacientes sin dolor y 2/7 con dolor leve a los 6 meses (Tablas 6 y 8 ).

El tiempo operatorio duración promedio de 120 min. El tiempo de hospitalización posquirúrgico tuvo un promedio de $48 \mathrm{~h}$ (Tabla 7).

Tabla 1. Resultados demográficos de población de estudio

\begin{tabular}{|cccccc|}
\hline Sexo & Frecuencia & $\begin{array}{c}\text { Edad } \\
\text { (promedio) }\end{array}$ & $\begin{array}{c}\text { Agresión por terceros } \\
\text { Aaída a nivel }\end{array}$ & $\begin{array}{c}\text { Etiología del trauma } \\
\text { Caccidente en bicicleta }\end{array}$ \\
\hline Femenino & 3 & 34 & 1 & 1 & 1 \\
\hline Masculino & 4 & 36 & 2 & $2(28,6 \%)$ & 1 \\
\hline Total & 7 & 35 & $3(42,8 \%)$ & $2(28,6 \%)$ \\
\hline
\end{tabular}


Tabla 2. Evolución de dinámica mandibular posquirúrgica de pacientes (en detalle) según índice de Helkimo

\begin{tabular}{|c|c|c|c|c|}
\hline Paciente & & 12 días & 3 meses & 6 meses \\
\hline \multirow[t]{4}{*}{1} & $\begin{array}{l}\text { Movilidad } \\
\text { mandibular }\end{array}$ & $\begin{array}{c}\text { Movilidad ligeramente reducida } \\
\text { (1) }\end{array}$ & Movilidad mandibular normal & $\begin{array}{c}\text { Movilidad mandibular normal } \\
(0)\end{array}$ \\
\hline & $\begin{array}{l}\text { Alteración en la } \\
\text { función articular }\end{array}$ & $\begin{array}{l}\text { No hay ruido ni desviación } \\
(0)\end{array}$ & $\begin{array}{l}\text { No hay ruido ni desviación } \\
(0)\end{array}$ & $\begin{array}{l}\text { No hay ruido ni desviación } \\
(0)\end{array}$ \\
\hline & $\begin{array}{l}\text { Dolor en movimiento } \\
\text { mandibular }\end{array}$ & Dolor referido a un solo movimiento & $\begin{array}{c}\text { No se presenta dolor } \\
(0)\end{array}$ & No se presenta dolor (0) \\
\hline & $\begin{array}{l}\text { Dolor articular } \\
\text { (ATM) }\end{array}$ & $\begin{array}{c}\text { Dolor a la palpación periauricular } \\
\text { unilateral o bilateral } \\
\text { de la articulación (1) }\end{array}$ & $\begin{array}{l}\text { No se presenta dolor } \\
\qquad(0)\end{array}$ & $\begin{array}{l}\text { No se presenta dolor } \\
\qquad(0)\end{array}$ \\
\hline \multirow[t]{4}{*}{2} & $\begin{array}{l}\text { Movilidad } \\
\text { mandibular }\end{array}$ & $\begin{array}{c}\text { Movilidad ligeramente reducida } \\
\text { (1) }\end{array}$ & $\begin{array}{c}\text { Movilidad ligeramente reducida } \\
\text { (1) }\end{array}$ & $\begin{array}{c}\text { Movilidad mandibular normal } \\
(0)\end{array}$ \\
\hline & $\begin{array}{l}\text { Dolor en movimiento } \\
\text { mandibular }\end{array}$ & $\begin{array}{l}\text { Dolor referido a dos o más } \\
\text { movimientos } \\
\text { (5) }\end{array}$ & $\begin{array}{l}\text { Dolor referido a un solo movimiento } \\
\text { (1) }\end{array}$ & $\begin{array}{l}\text { No se presenta dolor } \\
(0)\end{array}$ \\
\hline & Dolor muscular & $\begin{array}{c}\text { Dolor en } 1 \text { a } 3 \text { sitios } \\
\text { (1) }\end{array}$ & $\begin{array}{l}\text { Sin dolor } \\
(0)\end{array}$ & $\begin{array}{l}\text { Sin dolor } \\
(0)\end{array}$ \\
\hline & $\begin{array}{l}\text { Dolor articular } \\
\text { (ATM) }\end{array}$ & $\begin{array}{c}\text { Dolor a la palpación periauricular } \\
\text { unilateral o bilateral } \\
\text { de la articulación (1) }\end{array}$ & $\begin{array}{c}\text { Dolor a la palpación periauricular } \\
\text { unilateral o bilateral } \\
\text { de la articulación (1) }\end{array}$ & $\begin{array}{l}\text { No se presenta dolor } \\
\qquad(0)\end{array}$ \\
\hline \multirow[t]{2}{*}{3} & $\begin{array}{l}\text { Movilidad } \\
\text { mandibular }\end{array}$ & $\begin{array}{l}\text { Movilidad ligeramente reducida } \\
\text { (1) }\end{array}$ & Movilidad ligeramente reducida & $\begin{array}{c}\text { Movilidad mandibular normal } \\
(0)\end{array}$ \\
\hline & $\begin{array}{l}\text { Dolor articular } \\
\text { (ATM) }\end{array}$ & $\begin{array}{c}\text { Dolor a la palpación periauricular } \\
\text { unilateral o bilateral } \\
\text { de la articulación (1) }\end{array}$ & $\begin{array}{l}\text { No se presenta dolor } \\
(0)\end{array}$ & $\begin{array}{l}\text { No se presenta dolor } \\
(0)\end{array}$ \\
\hline \multirow[t]{5}{*}{4} & $\begin{array}{l}\text { Movilidad } \\
\text { mandibular }\end{array}$ & $\begin{array}{l}\text { Movilidad ligeramente reducida } \\
\text { (1) }\end{array}$ & Movilidad mandibular normal & $\begin{array}{c}\text { Movilidad mandibular normal } \\
(0)\end{array}$ \\
\hline & $\begin{array}{l}\text { Alteración en la } \\
\text { función articular }\end{array}$ & $\begin{array}{l}\text { No hay ruido ni desviación } \\
(0)\end{array}$ & $\begin{array}{l}\text { No hay ruido ni desviación } \\
\qquad(0)\end{array}$ & $\begin{array}{c}\text { No hay ruido ni desviación } \\
(0)\end{array}$ \\
\hline & $\begin{array}{l}\text { Dolor en movimiento } \\
\text { mandibular }\end{array}$ & $\begin{array}{l}\text { Dolor referido a un solo movimiento } \\
\text { (1) }\end{array}$ & $\begin{array}{l}\text { No se presenta dolor } \\
(0)\end{array}$ & $\begin{array}{l}\text { No se presenta dolor } \\
(0)\end{array}$ \\
\hline & Dolor muscular & $\begin{array}{c}\text { Dolor en } 1 \text { a } 3 \text { sitios } \\
\text { (1) }\end{array}$ & $\begin{array}{l}\text { No se presenta dolor } \\
(0)\end{array}$ & $\begin{array}{l}\text { No se presenta dolor } \\
(0)\end{array}$ \\
\hline & $\begin{array}{l}\text { Dolor articular } \\
\text { (ATM) }\end{array}$ & $\begin{array}{c}\text { Dolor a la palpación periauricular } \\
\text { unilateral o bilateral } \\
\text { de la articulación (1) }\end{array}$ & $\begin{array}{l}\text { No se presenta dolor } \\
(0)\end{array}$ & $\begin{array}{l}\text { No se presenta dolor } \\
(0)\end{array}$ \\
\hline
\end{tabular}


Tabla 2. Evolución de dinámica mandibular posquirúrgica de pacientes (en detalle) según índice de Helkimo (continuación)

\begin{tabular}{|c|c|c|c|c|}
\hline Paciente & & 12 días & 3 meses & 6 meses \\
\hline \multirow[t]{3}{*}{5} & $\begin{array}{l}\text { Movilidad } \\
\text { mandibular }\end{array}$ & $\begin{array}{l}\text { Movilidad ligeramente reducida } \\
\text { (1) }\end{array}$ & $\begin{array}{l}\text { Movilidad ligeramente reducida } \\
\text { (1) }\end{array}$ & Movilidad mandibular normal \\
\hline & $\begin{array}{l}\text { Dolor en movimiento } \\
\text { mandibular }\end{array}$ & $\begin{array}{l}\text { Dolor referido a un solo movimiento } \\
\text { (1) }\end{array}$ & $\begin{array}{l}\text { No se presenta dolor } \\
\qquad(0)\end{array}$ & $\begin{array}{l}\text { No se presenta dolor } \\
(0)\end{array}$ \\
\hline & $\begin{array}{l}\text { Dolor articular } \\
\text { (ATM) }\end{array}$ & $\begin{array}{c}\text { Dolor a la palpación periauricular } \\
\text { unilateral o bilateral de la } \\
\text { articulación (1) }\end{array}$ & $\begin{array}{l}\text { No se presenta dolor } \\
(0)\end{array}$ & $\begin{array}{l}\text { No se presenta dolor } \\
\qquad(0)\end{array}$ \\
\hline \multirow[t]{3}{*}{6} & $\begin{array}{l}\text { Movilidad } \\
\text { mandibular }\end{array}$ & $\begin{array}{l}\text { Movilidad ligeramente reducida } \\
\text { (1) }\end{array}$ & $\begin{array}{l}\text { Movilidad ligeramente reducida } \\
\text { (1) }\end{array}$ & $\begin{array}{l}\text { Movilidad mandibular normal } \\
(0)\end{array}$ \\
\hline & Dolor muscular & $\begin{array}{c}\text { Dolor en } 1 \text { a } 3 \text { sitios } \\
\text { (1) }\end{array}$ & $\begin{array}{l}\text { Dolor en } 1 \text { a } 3 \text { sitios } \\
\text { (1) }\end{array}$ & $\begin{array}{l}\text { No se presenta dolor } \\
(0)\end{array}$ \\
\hline & $\begin{array}{l}\text { Dolor articular } \\
\text { (ATM) }\end{array}$ & $\begin{array}{c}\text { Dolor a la palpación periauricular } \\
\text { unilateral o bilateral de la } \\
\text { articulación (1) }\end{array}$ & $\begin{array}{l}\text { No se presenta dolor } \\
(0)\end{array}$ & $\begin{array}{l}\text { No se presenta dolor } \\
(0)\end{array}$ \\
\hline \multirow[t]{2}{*}{7} & $\begin{array}{l}\text { Movilidad } \\
\text { mandibular }\end{array}$ & $\begin{array}{l}\text { Movilidad ligeramente reducida } \\
\text { (1) }\end{array}$ & Movilidad ligeramente reducida (1) & $\begin{array}{l}\text { Movilidad ligeramente } \\
\text { reducida (1) }\end{array}$ \\
\hline & $\begin{array}{l}\text { Alteración en la } \\
\text { función articular }\end{array}$ & $\begin{array}{l}\text { Ruido en ATM y/o desviación } \\
\geq 2 \mathrm{~mm} \text { (1) }\end{array}$ & $\begin{array}{l}\text { Ruido en ATM y/o desviación } \\
\geq 2 \mathrm{~mm} \text { (1) }\end{array}$ & $\begin{array}{l}\text { No hay ruido ni desviación } \\
\qquad(0)\end{array}$ \\
\hline
\end{tabular}

Tabla 3. Resultados de dinámica mandibular posquirúrgica según índice de Helkimo

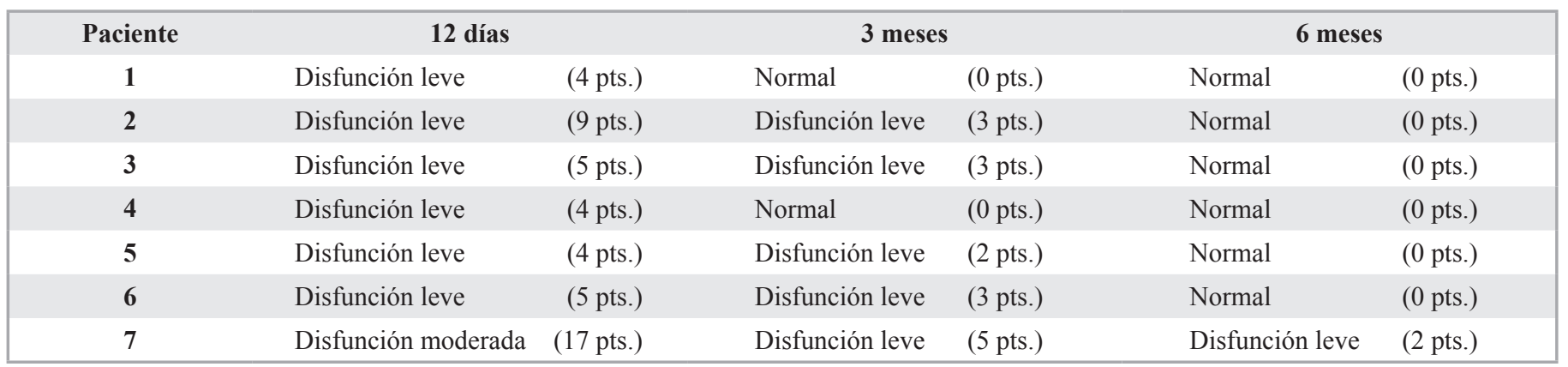


Tabla 4. Movilidad mandibular en pacientes pre y posquirúrgico

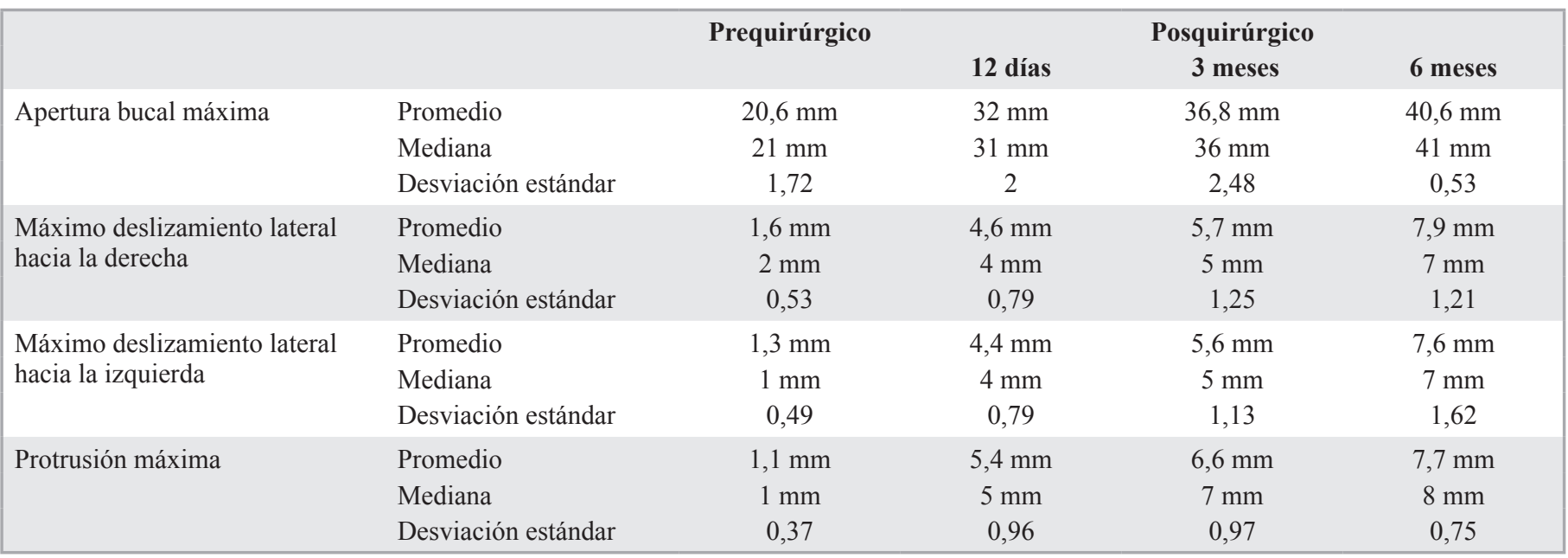

Tabla 5. Evaluación daño neurológico posoperatorio medido a través de la Escala de House-Brackmann

\begin{tabular}{|c|c|c|c|}
\hline Paciente & 12 días & 3 meses & 6 meses \\
\hline 1 & Grado I: Función facial normal & Grado I: Función facial normal & Grado I: Función facial normal \\
\hline 2 & Grado I: Función facial normal & Grado I: Función facial normal & Grado I: Función facial normal \\
\hline 3 & Grado II: Afectación discreta & Grado I: Función facial normal & Grado I: Función facial normal \\
\hline 4 & Grado I: Función facial normal & Grado I: Función facial normal & Grado I: Función facial normal \\
\hline 5 & Grado I: Función facial normal & Grado I: Función facial normal & Grado I: Función facial normal \\
\hline 6 & Grado I: Función facial normal & Grado I: Función facial normal & Grado I: Función facial normal \\
\hline 7 & Grado II: Afectación discreta & Grado II: Afectación discreta & Grado I: Función facial normal \\
\hline
\end{tabular}

Tabla 6. Evolución del dolor posoperatorio según Escala Visual Análoga (EVA)

\begin{tabular}{|c|c|c|c|}
\hline Paciente & 12 días & 3 meses & 6 meses \\
\hline 1 & 5/10 (Moderado) & 1/10 (Leve) & 0/10 (Sin dolor) \\
\hline 2 & 5/10 (Moderado) & 2/10 (Leve) & 0/10 (Sin dolor) \\
\hline 3 & 3/10 (Leve) & 2/10 (Leve) & 0/10 (Sin dolor) \\
\hline 4 & 6/10 (Moderado) & 3/10 (Leve) & 2/10 (Leve) \\
\hline 5 & 5/10 (Moderado) & 3/10 (Leve) & 0/10 (Sin dolor) \\
\hline 6 & 4/10 (Moderado) & 2/10 (Leve) & 0/10 (Sin dolor) \\
\hline 7 & 6/10 (Moderado) & 4/10 (Moderado) & 2/10 (Leve) \\
\hline
\end{tabular}

Tabla 7. Tiempo quirúrgico y de hospitalización posquirúrgico

\begin{tabular}{|lccc|}
\hline & Media & Mediana & \multicolumn{1}{c|}{ Desviación estándar } \\
\hline Tiempo quirúrgico & $120 \mathrm{~min}$ & $120 \mathrm{~min}$ & 4,08 \\
\hline Tiempo de hospitalización posquirúrgico & $48 \mathrm{~h}$ & $48 \mathrm{~h}$ & 1,41 \\
\hline
\end{tabular}


Tabla 8. Resultados dinámica mandibular, evolución neurológica y dolor posoperatorio

\begin{tabular}{|llccc|}
\hline \multirow{2}{*}{ Dinámica mandibular } & & $\mathbf{1 2}$ días & $\mathbf{3}$ meses & $\mathbf{6}$ meses \\
& Normal & 0 & $28,6 \%$ & $85,7 \%$ \\
& Disfunción leve & $85,7 \%$ & $71,4 \%$ & $14,3 \%$ \\
& Disfunción moderada & $14,3 \%$ & 0 & 0 \\
Evaluación neurológica & Grado I & $71,4 \%$ & $85,7 \%$ & $100 \%$ \\
& Grado II & $28,6 \%$ & $14,3 \%$ & 0 \\
\multirow{2}{*}{ Dolor } & Sin dolor & 0 & 0 & $71,4 \%$ \\
& Leve & $14,3 \%$ & $85,7 \%$ & $28,6 \%$ \\
\hline
\end{tabular}

Todos los pacientes recuperaron la oclusión que tenían de forma previa a la fractura mandibular. Las complicaciones posoperatorias registradas a los 30 y 90 días, según la clasificación de Clavien-Dindo ${ }^{12}$, fueron grado I en todos los pacientes operados. No se reportaron infecciones posoperatorias ni fístula salival. No se presentaron secuelas neurológicas tanto sensitivas como motoras asociadas al acto quirúrgico. No se reportaron casos que tuvieran la necesidad de reintervención quirúrgica.

\section{Discusión}

Las fracturas cóndilo mandibulares son las fracturas mandibulares de mayor prevalencia ${ }^{1}$, debido a que el cóndilo es una zona de menor resistencia mandibular ${ }^{13}$.

Su tratamiento es controversial y aún no existe consenso respecto a cuál alternativa terapéutica es la óptima ${ }^{3}$. Los métodos de tratamiento más comunes son reducción abierta y fijación interna mediante elementos de osteosíntesis (ORIF) y la reducción cerrada (CR) con fijación máxilo-mandibular (MMF). Según una revisión de Haug y Brandt ${ }^{14}$, en la cual se comparan ambas técnicas quirúrgicas, la reducción cerrada con MMF presenta mayor número de complicaciones, incluyendo entre ellas maloclusiones, asimetrías, dolor crónico, limitación en la dinámica mandibular y alteraciones en la posición condilar, mientras que la técnica de reducción abierta y fijación interna sólo presentaba como complicaciones una cicatriz visible y parálisis temporal del nervio facial con resolución posterior a los 6 meses.

La técnica de reducción cerrada con MMF presenta una tasa mayor de complicaciones de un 39\% en comparación con un 4\% de la técnica de ORIF ${ }^{4}$. Por otra parte, Hidding et al. ${ }^{15}$, compararon resultados de 5 años de estudio de estas técnicas y reporta- ron que un $64 \%$ de los pacientes que fueron tratados por reducción cerrada con MMF presentó desviación mandibular en apertura bucal, en comparación con un $10 \%$ en pacientes que fueron tratados con la técnica de reducción abierta y fijación interna. Haug y Assael $^{16}$, al comparar ambas técnicas, encontraron que después de 6 meses posteriores al tratamiento, los pacientes con reducción cerrada sufrían con más frecuencia dolor crónico, mientras que los pacientes bajo reducción abierta se quejaban de cicatrices perceptibles.

Se han desarrollado varias técnicas para perfeccionar la reducción abierta con fijación interna con el fin de disminuir las posibles complicaciones de secuelas neurológicas y de visibilidad de la cicatriz. Actualmente, la reducción y fijación óptima sumado a una función mandibular adecuada se han logrado mediante la asistencia de endoscopio cercano a la región de la fractura del cóndilo mandibular ${ }^{4}$. El endoscopio mejora la visibilidad para la reducción de los segmentos fracturados, reduciendo riesgo de lesión del nervio facial y visibilidad de la cicatriz ${ }^{17}$.

Nuestro estudio describe una técnica de reducción quirúrgica asistida por endoscopio, basada en un abordaje transoral a nivel de la rama mandibular para la reducción de los fragmentos fracturados asistida por endoscopio, sumado a un acceso extraoral retromandibular para la fijación de los fragmentos por elementos de osteosíntesis a placas y tornillos. Esta técnica no ha sido descrita previamente en la literatura, pero sí se han descrito otros estudios como Aboelatta et al. ${ }^{3}$, que describen una técnica que reduce los segmentos fracturados a través de una incisión intraoral a lo largo del borde anterior de la rama mandibular con la asistencia de endoscopio y una incisión submandibular adicional para facilitar la fijación de los fragmentos.

Un estudio de Handschel et al. ${ }^{18}$, demostró que el abordaje submandibular presentó peores resultados 
con respecto a la parálisis permanente del nervio facial y una cicatrización hipertrófica visible. Además, al comparar las cicatrices extraorales de los accesos submandibular, preauricular y retromandibular, las dos primeras presentaron un tamaño significativamente mayor que las del acceso retromandibular. En nuestro estudio no se presentaron secuelas neurológicas tanto sensitivas como motoras asociadas al acto quirúrgico, y la cicatriz localizada a nivel retromandibular era imperceptible.

Si bien la técnica que describimos posee dos accesos quirúrgicos a la rama mandibular (transoral y transparotídeo o retromandibular), lo que podría suponer una mayor morbilidad y/o mayor probabilidad de secuelas posquirúrgicas, no se reportaron algunas de las complicaciones descritas en las técnicas quirúrgicas convencionales para este tipo de fracturas.

Pacientes con maloclusión grave, posterior a un trauma, presentan mejores resultados con la reducción y fijación transoral abierta asistida por endoscopio para la prevención de trastornos oclusales, según indica un estudio prospectivo de Kokemueller et al. ${ }^{19}$, estudio que coincide con nuestros resultados, los que fueron favorables tanto para la oclusión como para la dinamia mandibular.

La reducción quirúrgica asistida endoscópicamente, requiere una mayor curva de aprendizaje por parte del equipo quirúrgico ${ }^{20-22}$. En la literatura, la duración media de la operación es variable. En un estudio de Kang et al. ${ }^{4}$, la duración media de la operación fue de 200 min, González-García et al. ${ }^{22}$, informaron que la duración media de la operación fue de 81 min. Akdag et al. ${ }^{23}$, tuvieron una duración promedio de 106 min. Nuestro estudio desarrolló sus tratamientos quirúrgicos en un tiempo promedio de $120 \mathrm{~min}$, considerando un abordaje retromandibular extraoral adicional. Sin embargo, es muy difícil establecer un estándar ya que los tiempos de operación se calculan de diversas maneras.

Los pacientes operados con nuestra técnica descrita presentaron mejorías significativas en los hallazgos clínicos registrados después de la operación; registraron una apertura en rangos normales posoperatorios. No se evidenciaron daños neurológicos sensitivos ni motores.

La técnica presentada minimiza las desventajas de las técnicas conocidas. La asistencia de endoscopio reduce las posibles complicaciones de ambos accesos quirúrgicos y del procedimiento propiamente tal.

El uso de endoscopio permite una mejor visibilidad de las estructuras, facilita la reducción de los segmentos fracturados o en su defecto, permite posicionarlos en la cara lateral de la rama mandibular, lugar anatómico de fácil acceso desde el punto de vista quirúrgico.

Dentro de las limitaciones de nuestro estudio está el tamaño de la muestra, al tratarse de siete pacientes.

Se sugiere realizar la técnica presentada en un mayor número de pacientes que permitan futuras líneas de investigación sobre la técnica.

Como ha sido reportado en la literatura, el abordaje transoral disminuye los riesgos de lesión al nervio facial y visibilidad de una cicatriz extraoral, pero al ser un acceso intraoral, existe una limitación de movimientos y visualización del campo operatorio al momento de fijar e instalar los elementos de osteosíntesis en los fragmentos fracturados, de esta manera adicionamos un abordaje retromandibular extraoral para poder llevar a cabo la fijación por medio de elementos de osteosíntesis a placas y tornillos.

\section{Conclusión}

La técnica quirúrgica de reducción asistida por endoscopio para las fracturas de cóndilo mandibular ofrece un complemento a la técnica quirúrgica convencional.

La visión de los segmentos fracturados por el uso del endoscopio favorece la reducción y una menor manipulación quirúrgica extraoral, lo que determinaría menores complicaciones asociadas a la técnica quirúrgica convencional.

Se requieren nuevos estudios comparativos para establecer de forma objetiva las ventajas que tendría este complemento quirúrgico a la técnica quirúrgica convencional.

\section{Responsabilidades éticas}

Protección de personas y animales. Los autores declaran que para esta investigación no se han realizado experimentos en seres humanos ni en animales.

Confidencialidad de los datos. Los autores declaran que en este artículo no aparecen datos de pacientes.

Conflictos de interés: no hay. 


\section{Bibliografía}

1. Blumer M, Guggenbühl T, Wagner MEH, Rostetter C, Rücker M, Gander T. Outcome of Surgically Treated Fractures of the Condylar Process by an Endoscopic Assisted Transoral Approach. J Oral Maxillofac Surg. 2019;77:133.e1-133.e9. doi: 10.1016/j.joms.2018.08.013. Epub 2018 Aug 24.

2. Mannion CJ, Loukota RA. Endoscopically assisted treatment of condylar fracturesexperiences following an educational course. Br J Oral Maxillofac Surg. 2010;48:453-4.

3. Aboelatta YA, Elbarbary AS, Abdelazeem S, Massoud KS, Safe II. Minimizing the Submandibular Incision in Endoscopic Subcondylar Fracture Repair. Craniomaxillofac Trauma Reconstr. 2015;8:315-20.

4. Kang SH, Choi EJ, Kim HW, Kim HJ, Cha IH, Nam W. Complications in endoscopicassisted open reduction and internal fixation of mandibular condyle fractures. Oral Surg Oral Med Oral Pathol Oral Radiol. 2012;113:201-6.

5. Arcuri F, Brucoli M, Baragiotta N, Benech R, Ferrero S, Benech A. Analysis of complications following endoscopically assisted treatment of mandibular condylar fractures. J Craniofac Surg. 2012;23:e1968.

6. Hwang NH, Lee YH, You HJ, Yoon ES, Kim DW. Endoscope-Assisted Transoral Fixation of Mandibular Condyle Fractures: Submandibular Versus Transoral Endoscopic Approach. J Craniofac Surg. 2016;27:1170-4.

7. Ellis E 3rd, Simon P, Throckmorton GS. Occlusal results after open or closed treatment of fractures of the mandibular condylar process. J Oral Maxillofac Surg. 2000;58:260-8.
8. Bhagol A, Singh V, Kumar I, Verma A. Prospective evaluation of a new classification system for the management of mandibular subcondylar fractures. J Oral Maxillofac Surg. 2011;69:1159-65.

9. Suhas S, Ramdas S, Lingam PP, Naveen Kumar HR, Sasidharan A, Aadithya R. Assessment of temporomandibular joint dysfunction in condylar fracture of the mandible using the Helkimo index. Indian J Plast Surg. 2017;50:207-12.

10. Devèze A, Ambrun A, Gratacap M, Céruse P, Dubreuil C, Tringali S. Parálisis facial periférica. EMC - Otorrinolaringología 2013;42:1-24 [Artículo E - 20-260-A-10].

11. Pardo C, Muñoz T, Chamorro C. Monitorización del dolor: Recomendaciones del grupo de trabajo de analgesia y sedación de la SEMICYUC. Medicina Intensiva 2006;30:379-85.

12. Dindo D, Demartines N, Clavien PA. Classification of surgical complications: a new proposal with evaluation in a cohort of 6336 patients and results of a survey. Ann Surg. 2004;240:205-13.

13. Hupp J, Ellis E, Tucker M. Contemporary Oral and Maxillofacial Surgery. 6th ed. Mosby 2013

14. Haug RH, Brandt MT. Closed reduction, open reduction, and endoscopic assistance: current thoughts on the management of mandibular condyle fractures. Plast Reconstr Surg. 2007;120(7 Suppl 2):90S-102S

15. Hidding J, Wolf R, Pingel D. Surgical versus non-surgical treatment of fractures of the articular process of the mandible. $\mathrm{J}$ Craniomaxillofac Surg. 1992;20:345-7.

16. Haug RH, Assael LA. Outcomes of open versus closed treatment of mandibular subcondylar fractures. J Oral Maxillofac Surg. 2001;59:370-5.

17. Schön R, Schramm A, Gellrich NC, Schmelzeisen R. Follow-up of condylar fractures of the mandible in 8 patients at 18 months after transoral endoscopicassisted open treatment. J Oral Maxillofac Surg. 2003;61:49-54.

18. Handschel J, Rüggeberg T, Depprich R, Schwarz F, Meyer U, Kübler NR, et al. Comparison of various approaches for the treatment of fractures of the mandibular condylar process. J Craniomaxillofac Surg. 2012;40:e397-401.

19. Kokemueller H, Konstantinovic VS, Barth EL, Goldhahn S, von See C, Tavassol F, et al. Endoscope-assisted transoral reduction and internal fixation versus closed treatment of mandibular condylar process fractures--a prospective doublecenter study. J Oral Maxillofac Surg. 2012;70:384-95.

20. Neff A, Chossegros C, Blanc JL, Champsaur P, Cheynet F, Devauchelle B, et al., Position paper from the IBRA Symposium on Surgery of the Head--the 2nd International Symposium for Condylar Fracture Osteosynthesis, Marseille, France 2012. J Craniomaxillofac Surg. 2014:42:1234-49.

21. Kellman RM, Cienfuegos R. Endoscopic approaches to subcondylar fractures of the mandible. Facial Plast Surg. 2009;25:23-8.

22. González-García R, Sanromán JF, Goizueta-Adame C, Rodríguez-Campo FJ, Cho-Lee GY. Transoral endoscopicassisted management of subcondylar fractures in 17 patients: an alternative to open reduction with rigid internal fixation and closed reduction with maxillomandibular fixation. Int J Oral Maxillofac Surg. 2009;38:19-25.

23. Akdag O, Yildiran G, Abaci M, Tosun Z. Endoscopic-Assisted Treatment Combined With Transoral and Transbuccal Approach to Mandibular Subcondylar Fractures. J Oral Maxillofac Surg. 2018;76:831.e1831.e5. 